\title{
Clinical Presentation and Therapy of Primary Immune Thrombocytopenia Resistant to Splenectomy
}

\author{
Aleksandar Kara-Jovanović, ${ }^{1}$ Nada Suvajdžić-Vuković. ${ }^{2}$
}

\begin{abstract}
Background: A satisfactory therapeutic response is achieved with splenectomy in 60-80\% of the patients diagnosed with immune thrombocytopenic purpura (ITP). There is an ongoing consensus on the short-term efficacy of splenectomy, however, its long-term efficacy remains controversial. Our aim was to establish the frequency of resistance and relapse after splenectomy, the occurrence of complications, the therapeutic strategies and the drug efficacy in splenectomy-resistant ITP. Methods: We retrospectively analyzed 138 adult ITP patients who had been previously diagnosed and treated at the Clinic of Hematology, Clinical Center of Serbia, and who underwent splenectomy between 1987 and 2018. Results: Of the 138 ITP patients, 20.3\% ( $\mathrm{n}=28 / 138$ ) were refractory to splenectomy, $11.6 \%(n=16 / 138)$ relapsed and $8.7 \%(n=12 / 138)$ were primarily resistant. The average post-splenectomy follow-up period was 117 months (range 3-474). The average follow-up period of the patients resistant to splenectomy was 147 months (range 23-474). Of the patients refractory to splenectomy, $67.8 \%(n=19 / 28)$ showed a good therapeutic response: $49 \%(n=14 / 28)$ complete remission and $18.8 \%(n=5 / 28)$ partial remission. The response was usually achieved using the following drugs: romiplostim (100\%), eltrombopag (75\%), cyclosporine (66.67\%), mycophenolate mofetil (50\%), danazol (50\%) and corticosteroids (40.9\%). Hemorrhagic and non-hemorrhagic complications occurred in $78.6 \%(n=22 / 28)$ and $28.6 \%$ ( $n=8 / 28)$ of the patients, respectively. Conclusion: Splenectomy remains a very efficient therapeutic modality for the treatment of ITP patients with a high percentage of splenectomy-resistant patients achieving remission. Thrombopoietin receptor agonists have shown exceptional results so far in the treatment of refractory ITP patients.
\end{abstract}

Key Words: Immune thrombocytopenia; Idiopathic thrombocytopenic purpura; Refractory immune thrombocytopenia, Thrombopoietin receptor agonists (Source: MeSH-NLM).

\section{Introduction}

Immune thrombocytopenic purpura (ITP) is an immune-mediated acquired disease of adults and children characterized by a transient or persistent decrease of the platelet count below $100 \times 10^{\circ} / \mathrm{L}$ in the absence of evident predisposing factors, and by an increased risk of bleeding. ${ }^{1-2}$ The incidence of primary ITP in adults is 3.3/100 000 adults per year with a prevalence of 9.5 per 100000 adults. ${ }^{1}$ In 2009, the International Working Group (IWG) presented standards for definitions, classification criteria, and outcome measures. The classification scheme included division by disease phase: newly diagnosed ITP ( $<3$ months), persistent ITP (3-12 months), and chronic ITP ( $>12$ months). Refractory ITP is defined as a disease that does not respond to or relapses after splenectomy and that requires treatment to reduce the risk of clinically significant bleeding. ${ }^{1}$

The clinical presentation of ITP is most often asymptomatic or with a varying bleeding tendency. The severity of bleeding frequently correlates with a low platelet count and older age. However, many patients with ITP do not experience significant bleedings despite having severe thrombocytopenia.3-4 Mucocutaneous bleeding (epistaxis, menorrhagia, gingival and gastrointestinal bleeding) is the most common clinical manifestation of ITP. Intracranial hemorrhage is the most worrisome complication of ITP, with an incidence of 6/340 adults newly diagnosed with ITP. 5 Patients with ITP have an increased risk of thrombotic events in comparison to adults without ITP, as well as fatigue, infections, hematologic malignancies and reduced quality of life. ${ }^{5}$
The diagnosis of ITP is made when all other causes of thrombocytopenia are ruled out.

The therapy objectives are to achieve a safe number of platelets (often $>30 \times 10^{9} / \mathrm{L}$ ), to avoid life-threatening bleeding and long-term treatment, and to sustain a normal quality of life. ' The therapy should be tailored according to the clinical presentation, presence of additional bleeding risk factors (such as anticoagulant therapy, dialysis, surgical procedures), comorbidities, individual side effect tolerance, as well as the platelet count. Treatment should begin when the platelet count is $<30 \times 10^{9} / \mathrm{L}$, or when bleeding symptoms present in spite of a high thrombocyte count (Table 1) . $^{6-7}$

Splenectomy is reserved for patients unresponsive to or intolerant to corticosteroids. By performing splenectomy, antibody-coated platelets are no longer removed via the spleen and spleen antiplatelet antibodies are no longer produced. Splenectomy should be postponed, if possible, for 6-12 months, considering a possible spontaneous or therapeutically induced remission. ${ }^{1,68-9}$ Good therapeutic response is achieved in 60$80 \%$ of patients with ITP. ${ }^{8}$ Even though there is a consensus regarding the short-term efficacy of splenectomy, findings on its long-term efficacy and side-effects are controversial. Particular studies show that splenectomy leads to long-term remission in $60-80 \%$ of patients, while other studies imply that all patients with ITP relapse after a sufficiently long follow-up period. In most published studies, remission is achieved in $60 \%$ of patients, with $75 \%$ of relapses recorded in the first 4 years. The response was achieved in $83 \%$ of patients who needed further

\footnotetext{
Medical Faculty, University in Belgrade, Belgrade, Serbia.

2 Clinic of Hematology, Clinical Center of Serbia in Belgrade, Serbia.
}

About the Author: Aleksandar Kara-Jovanović is currently a 6th-year medical student in the Faculty of Medicine at the University of Belgrade, Serbia within the six-year integrated academic studies. He is currently working on a research paper concerning thrombotic events in acute myeloid leukemia.

Correspondence:

Aleksandar Kara-Jovanović

Address: 8 Dr Subotića Street, Belgrade, Serbia.

Email: aleksandar.karajovanovic@gmail.com
Editor: Mihnea-Alexandru Căman Student Editors: David Avelar Rodriguez Submission: Dec 10, 2019 Acceptance: Dec 28, 2019 Publication: Apr 30, 2020 Process: Peer-reviewed 
treatment after splenectomy, which implied that splenectomy can convert a drug-resistant patient to a patient with a good response. ${ }^{10}$ Currently, there are many therapeutic modalities for patients resistant to splenectomy. Approximately $75 \%$ of patients with refractory ITP eventually achieve remission, although it occurs slowly. Patients who do not achieve remission instead experience an extremely difficult disease course with significant morbidity and mortality rates. It is possible that newer forms of treatment, such as rituximab, thrombopoietic factors, and monoclonal antibodies, may alter the outcomes for this extremely resistant subgroup. ${ }^{11}$

The aim of our study was to establish the frequency of resistance and relapse after splenectomy, the occurrence of bleeding, infections and thrombotic events, the therapeutic strategies and the drug efficacy in a group of patients with splenectomy-resistant ITP.

Table 1. The Therapy of Immune Thrombocytopenia (ITP). ${ }^{6}$

\begin{tabular}{l|l}
$\begin{array}{l}\text { First-line } \\
\text { management } \\
\text { (initial treatment) }\end{array}$ & $\begin{array}{l}\text { - corticosteroids (prednisone, methylprednisolone, } \\
\text { dexamethasone) } \\
\bullet\end{array}$ \\
\hline \multirow{2}{*}{$\begin{array}{l}\text { Second-line } \\
\text { management }\end{array}$} & $\begin{array}{l}\text { - splenectomy } \\
\text { - azathioprine; cyclosporine A; vinca alkaloids; } \\
\text { cyclophosphamide; thrombopoietin receptor agonist (TP0- } \\
\text { RAs); mycophenolic acid; danazol; dapsone; rituximab }\end{array}$ \\
\hline \multirow{3}{*}{$\begin{array}{l}\text { Third-line } \\
\text { management } \\
\text { (for refractory ITP) }\end{array}$} & $\begin{array}{l}\text { Category A: } \\
\text { - thrombopoietin receptor agonists (TPO-RAs) }\end{array}$ \\
\cline { 2 - 2 } & $\begin{array}{l}\text { Category B: } \\
\text { - combination of medications from the } 1^{\text {st }} \text { and } 2^{\text {nd }} \text { line } \\
\text { management }\end{array}$
\end{tabular}

\section{Patients and Methods}

We retrospectively analyzed 138 adult patients with ITP who had been previously diagnosed and treated at the Clinic of Hematology, Clinical Center of Serbia, and who underwent splenectomy between 1987 and 2018. The following laboratory tests were run for each patient: blood tests - erythrocyte, leukocyte and platelet (PLT) counts, hemoglobin value, and leukocyte formula, hemostasis tests - fibrinogen, prothrombin time (PT), activated partial thromboplastin time (aPTT), morphological and cytochemical analysis of the bone marrow, thyroid status, abdominal ultrasonography and platelet kinetics (in most patients). Splenectomy was indicated in cortico-resistant (PLT<30x109/L) or cortico-sensitive patients. In order to attain the recommended platelet count for splenectomy $\left(P L T>50 \times 10^{9} / \mathrm{L}\right)$, patients were premedicated with corticosteroids, intravenous immunoglobulins (IVIg) and platelet transfusions. A "Complete response" (CR) is defined by the IWG as any platelet count of at least $150 \times 10^{\circ} / \mathrm{L}$. "Partial response" (PR) is defined as any platelet count $>50 \times 10^{\circ} / \mathrm{L}$. Refractory ITP is defined as any platelet count $<30 \times 10^{\circ} / \mathrm{L}$. Relapse is defined as any platelet count $<150 \times 10^{9} / \mathrm{L}$ in patients who previously achieved a CR or $50 \times 10^{9} / \mathrm{L}$ in patients who previously achieved a PR. ${ }^{2}$ The software used for the statistical analysis was "Microsoft Office Excel 2007"

\section{Results}

In this retrospective study, we analyzed 138 patients with adult ITP who had been previously diagnosed, treated and splenectomized at the Clinic of Hematology, Clinical Center of Serbia, from 1983 to 2018. From 138 patients who were splenectomized, $28(20.3 \%)$ were refractory to splenectomy. Of these, $16(11.6 \%)$ relapsed and $12(8.7 \%)$ had no response (Figure 1). The average follow-up period after splenectomy was 117 months $(3-474)$. Only one patient $(0.79 \%)$ was observed for less than a year. We observed $37.8 \%(n=52 / 138)$ of the patients for longer than 5 years, and $40 \%(n=55 / 138)$ for more than 10 years.

Hemorrhagic syndrome developed in $22 / 28$ (78.6\%) patients. Patients most commonly had hematomas, petechiae, and ecchymoses $(61 \%$, $n=17 / 28)$, epistaxes and gingival bleeding $(18 \%, n=5 / 28)$, menorrhagias and metrorrhagias $(18 \%, n=5 / 28)$. There was no reported intracranial hemorrhage. Incidence and localization of bleeding are shown in Figure 3.
Non-hemorrhagic complications or other comorbidities were recorded in 8 (28.6\%) patients: Non-Hodgkin's lymphoma $(2 / 28,7,1 \%)$, acute renal failure $(1 / 28,3.6 \%)$, systemic lupus erythematosus $(1 / 28,3,6 \%)$, abscesses $(2 / 28,7.1 \%)$ or infections $(3 / 28,10.7 \%)$. There were no reported death outcomes.

Figure 1. Patient Distribution Relative to Splenectomy Response.

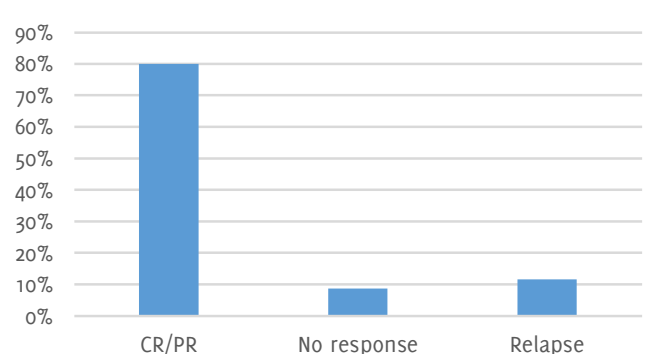

Legend: $C R=$ complete response. $P R=$ partial response.

The average follow-up period of the patients resistant to splenectomy was 147 months (23-474). Most relapses occurred in the first year after splenectomy $(6 / 28,21.4 \%)$, while in one patient $(1 / 28,3.6 \%)$ relapse occurred after 18 years (Figure 2). Laparotomy was performed in $64 \%$ $(n=18 / 28)$ of the patients, and laparoscopy was performed in $36 \%$ $(n=10 / 28)$ of the patients, with no significant differences in response. of the patients refractory to splenectomy, $67.8 \%(n=19 / 28)$ showed a good therapeutic response: $49 \%(n=14 / 28)$ complete remission and $18.8 \%(n=5 / 28)$ partial remission.

Figure 2. Relapse Incidence after Splenectomy.

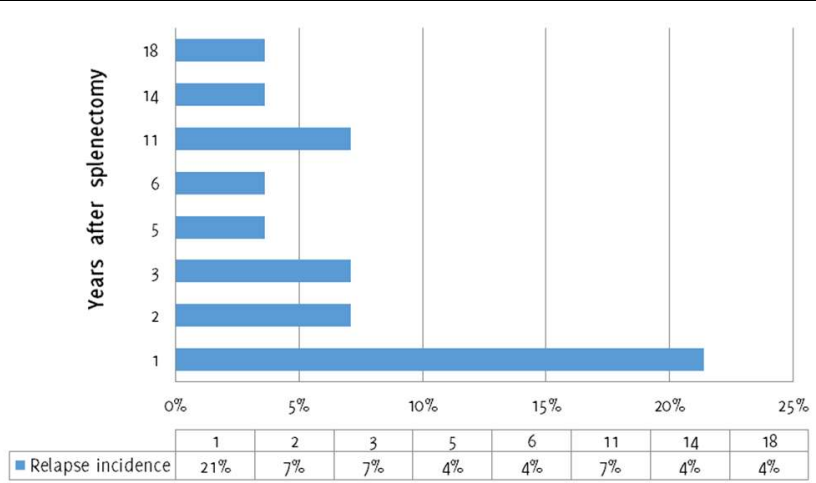

Figure 3. Incidence and Localization of Bleeding.

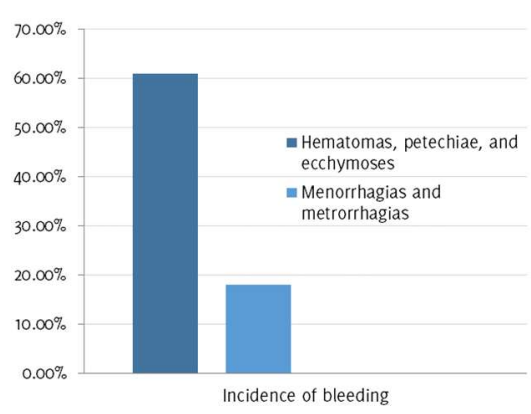

Out of the 28 splenectomized patients with refractory ITP, 27 patients underwent further treatment. Therapeutic lines and modalities are shown in Figure 4, and the therapeutic effect is shown in Figure 5. Prednisone was used in 22 patients $(81.5 \%$ ) and lead to a good therapeutic response (CR+PR) in 9 patients $(40.9 \%)$. Romiplostim was used in 2 patients (7.4\%) who achieved a good response (100\%). Eltrombopag was used in 4 patients $(14.81 \%)$, leading to a good 
therapeutic response in 3 patients $(75 \%)$. Cyclosporine was used in 3 patients $(11.1 \%)$, leading to a good therapeutic response in 2 patients $(66.7 \%)$. Mycophenolic acid was used in 5 patients (18.5\%), leading to a good therapeutic response in 3 patients (60\%). Danazol was used in 6 patients $(22.2 \%)$, leading to a good therapeutic response in 3 patients $(50 \%)$. Azathioprine was used in 11 patients $(40.7 \%)$, leading to a good therapeutic response in 4 patients $(36.4 \%)$. Cyclophosphamide was used in 4 patients $(14.8 \%$ ) with only one patient $(25 \%)$ achieving a good therapeutic response. Dapsone was used in one patient $(3.7 \%)$ without response. Excision of an accessory spleen was performed in one patient (3.7\%) without effect. Vinca alkaloids were prescribed in 5 patients $(18.5 \%)$, with only one patient $(20 \%)$ achieving a good therapeutic response.

Figure 4. Distribution of Patients based on the Employed Therapeutic Modalities after Splenectomy.

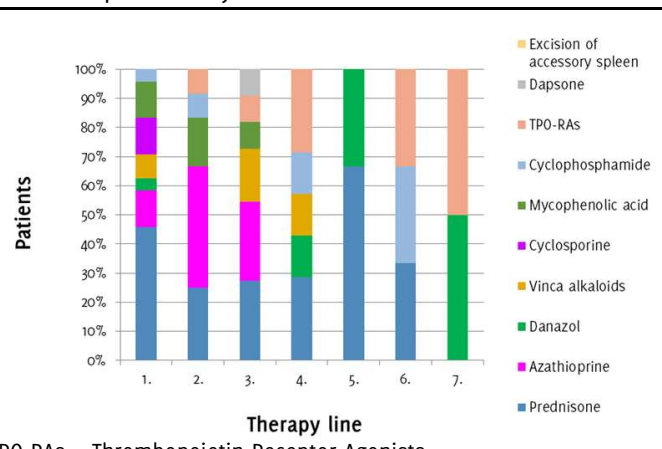

Legend: TPO-RAs $=$ Thrombopoietin Receptor Agonists.

Figure 5. The Effect of Therapy.

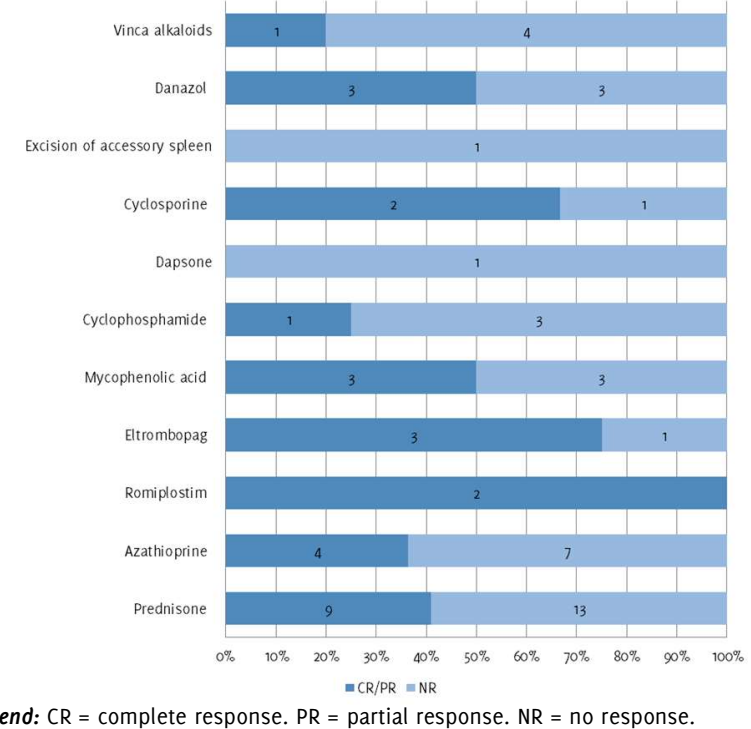

\section{Discussion}

Our study included 138 adult patients with ITP, out of whom $20.3 \%$ $(n=28 / 138)$ were refractory to splenectomy. Relapse occurred in $11.6 \%$ $(n=16 / 138)$ of patients, whilst $8.7 \%(n=12 / 138)$ of patients had no response to splenectomy. The average follow-up period of patients resistant to splenectomy was 147 months (23-474). Most relapses occurred in the first year after splenectomy $(n=6 / 28,21.4 \%)$, while in one patient $(3.6 \%)$ relapse occurred after 18 years. The study of Mcmillan et al., which involved 105 ITP patients refractory to splenectomy, reports a frequent occurrence of relapse $(66 \%)$, most often in the first 3 months after splenectomy ( $45 \%$ of patients), and no response in $22 \%$ of cases after splenectomy was performed." Vianelli et al., who studied 233 splenectomized patients, reports a relapse rate of $75 \%$ (after 48 months) and no response in $40 \%$ of cases. ${ }^{12}$ In the retrospective study of Ahmed et al., involving 167 patients, relapse occurred in $30 \%$ of the cases (after 54 months) and $14 \%$ of the patients experienced no response. ${ }^{13}$

After splenectomy, our study reported a good therapeutic response with different therapeutic modalities in $67.8 \%$ of the patients: CR $49 \%$ and PR $18.8 \%$, similarly to Mcmillan's study (71.4\%)." A stable therapeutic response was achieved with the following medications: romiplostim $(2 / 2,100 \%)$, eltrombopag $(3 / 4,75 \%)$, cyclosporine $(2 / 3$, $66.67 \%)$, mycophenolic acid $(3 / 6,50 \%)$, danazol $(3 / 6,50 \%)$, prednisone $(9 / 22,40.9 \%)$. Also, $29.6 \%(8 / 27)$ of the patients had no response to therapy. Mcmillan et al. showed that a stable therapeutic response was achieved with following medications: danazol (33.9\%), cyclophosphamide $(25.7 \%)$, prednisone (19\%), and azathioprine (15.8\%); $28 \%$ of the patients did not respond to therapy. The percentage of patients resistant to all therapeutic modalities is similar in both studies." However, Mcmillan's study does not take TPO-RAs into consideration, which may be the reason for the discrepancy between our studies. In the study of Saleh et al., 299 patients (115 splenectomized) were treated for 3 years with eltrombopag. Cood therapeutic effect was noted in $80 \%(92 / 115)$ of the splenectomized patients, as well as in $50 \%$ of the multi-resistant patients (treated with more than 4 therapeutic lines). The effect was maintained for 2 years. ${ }^{14}$ In the research of Kuter et al., 292 ITP patients (95 splenectomized) were treated with romiplostim for 5 years with a good therapeutic effect in $67 \%(64 / 95)$ of the splenectomized patients. The effect was maintained for 2 years also. ${ }^{15}$ Consequently, the results of our study coincide with results of Saleh et al., as well as with the results of Kuter et al. Our study reported hemorrhagic syndromes in $22 / 28(78.6 \%)$ of the patients: hematomas, petechiae, ecchymoses $(61 \%)$, epistaxes and gingival bleedings $(18 \%)$, menorrhagias and metrorrhagias (18\%), with no intracranial hemorrhage reported. Non-hemorrhagic complications or comorbidities were seen in $8(28.6 \%)$ of the patients: Non-Hodgkin's lymphoma $2 / 28(7.1 \%)$, acute renal failure $1 / 28(3.6 \%)$, systemic lupus erythematosus $1 / 28(3.6 \%)$, abscesses $2 / 28(7.1 \%)$ and infections $3 / 28$ (10.7\%). There were no reported death outcomes. Mcmillan et al. did not describe morbidity, but only a mortality regarding hemorrhagic events of $10 \%$ (intracranial hemorrhage $90 \%$ ), malignancy and cardiovascular disease $13.9 \%$. Other noted complications/comorbidities in Mcmillan's study were deep venous thrombosis ( $12.4 \%$ ), systemic lupus erythematosus (2.9\%), Non-Hodgkin's lymphoma (1.9\%) and Hodgkin's lymphoma (2.9\%). ${ }^{11}$ The study of Saleh et al reported a lower incidence of hemorrhagic complications after the use of eltrombopag (from $56 \%$ to $11 \%$ during the next 3 years), thromboembolic events $(4 \%)$ and elevation of liver enzymes $(2 \%) .{ }^{14}$ The study of Kuter et al, reported hemorrhagic complications in $57 \%$ of the patients: hematomas and petechiae $(32 \%)$, epistaxes and gingival bleeding $(41 \%) .{ }^{15}$ In comparison, our study had a higher incidence of complications, but without death outcomes.

\section{Conclusion}

Splenectomy represents a very efficient therapeutic modality for patients with ITP. Patients who remained resistant after splenectomy had a higher rate of CR. In our study, $20.3 \%$ of the patients were refractory to splenectomy, out of whom $11.6 \%$ relapsed (with the highest incidence in the first year after splenectomy, $21.4 \%$ ), and $8.7 \%$ were resistant to splenectomy. Our research showed that $67.8 \%$ of the patients refractory to splenectomy achieved a good therapeutic response ( $49 \%$ CR and $18.8 \%$ PR). A stable therapeutic response was achieved with the following medications: romiplostim (100\%), eltrombopag $(75 \%)$, cyclosporine $(66.67 \%)$, mycophenolic acid $(50 \%)$, danazol (50\%), prednisone $(40.9 \%)$. Thus, thrombopoietin receptor agonists exhibited the greatest success so far in treating patients with refractory ITP. The limitations of our study were a small sample of patients with a resistant form of ITP treated with thrombopoietin receptor agonists, as well as a heterogeneous follow-up period after splenectomy. 


\section{References}

1. Lambert MP, Gernsheimer TB. Clinical updates in adult immune thrombocytopenia. Blood. 2017 May 25;129(21):2829-35

2. Rodeghiero F, Stasi R, Gernsheimer T, Michel M, Provan D, Arnold DM, et al. Standardization of terminology, definitions and outcome criteria in immune thrombocytopenic purpura of adults and children: report from an international working group. Blood. 2009 Mar 12;113(11):2386-93.

3. Portielje JE, Westendorp RG, Kluin-Nelemans HC, Brand A. Morbidity and mortality in adults with idiopathic thrombocytopenic purpura. Blood. 2001 May 1;97(9):2549-54

4. Cohen YC, Djuibegovic B, Shamai-Lubovitz 0, Mozes B. The bleeding risk and natural history of idiopathic thrombocytopenic purpura in patients with persistent low platelet counts. Arch Intern Med. 2000 Jun 12;160(11):1630-8.

5. Kistanguri G, McCrae KR. Immune Thrombocytopenia. Hematol Oncol Clin North Am. 2013 Jun;27(3):495-520.

6. Provan D, Stasi $R$, Newland AC, Blanchette VS, Bolton-maggs $P$, James $B$, et al. International consensus report on the investigation and management of primary immune thrombocytopenia International consensus report on the investigation and management of primary immune thrombocytopenia. Blood. 2010 Jan 14;115(2):168-86.

7. British Committee for Standards in Haematology General Haematology Task Force. Guidelines for the investigation and management of idiopathic thrombocytopenic purpura in adults, children and in pregnancy. $\mathrm{Br} J$ Haematol. 2003 Feb; $120(4): 574-96$

8. Chaturvedi S, Arnold DM, McCrae KR. Splenectomy for immune thrombocytopenia: Down but not out. Blood. 2018 Mar 15;131(11):1172-82.
9. Neunert C, Lim W, Crowther M, Cohen A, Solberg L Jr, Crowther MA, American Society of Hematology. The American Society of Hematology 2011 evidence-based practice guideline for immune thrombocytopenia. Blood. 2011 Apr 21;117(16):4190-207.

10. Vianelli N, Galli M, De Vivo A, Intermesoli T, Giannini B, Mazzucconi MG, et al. Efficacy and safety of splenectomy in immune thrombocytopenic purpura: Longterm results of 402 cases. Haematologica. 2005;90(1):72-7.

11. Mcmillan R, Durette C. Long-term outcomes in adults with chronic ITP after splenectomy failure. Blood. 2004 Aug 15;104(4):956-60.

12. Vianelli N, Palandri F, Polverelli N, Stasi R, Joelsson J, Johansson E, et al. Splenectomy as a curative treatment for immune thrombocytopenia: a retrospective analysis of 233 patients with a minimum follow up of 10 years. Haematologica. 2013 Jun;98(6):875-80.

13. Ahmed R, Devasia AJ, Viswabandya A, Lakshmi KM, Abraham A, Karl S, et al Long-term outcome following splenectomy for chronic and persistent immune thrombocytopenia (ITP) in adults and children: Splenectomy in ITP. Ann Hematol. 2016 Sep;95(9):1429-34.

14. Saleh MN, Bussel JB, Cheng G, Meyer O, Bailey CK, Arning M. Safety and efficacy of eltrombopag for treatment of chronic immune thrombocytopenia: results of the long-term, open-label EXTEND study. Blood. 2013 Jan 17;121(3):537-45.

15. Kuter DJ, Bussel JB, Newland A, Baker Rl, Lyons M, Wasser J, et al. Long-term treatment with romiplostim in patients with chronic immune thrombocytopenia: safety and efficacy. Br J Haematol. 2013 May; $161(3): 411-23$.

\section{Acknowledgments}

The abstract of the paper was orally presented at the XIV Young European Scientist - Meeting, Porto, Portugal, 09-2019, and accepted for presentation at the XII International Congress on Autoimmunity, Athens, Greece, 20-05-2020.

Conflict of Interest Statement a Funding

The Authors have no funding, financial relationships or conflicts of interest to disclose.

\section{Author Contributions}

Conceptualization: NS. Methodology: AK, and NS. Software: AK. Validation: NS. Formal Analysis: AK. Data Curation: NS. Investigation: AK. Writing Original Draft: AK. Writing - Review Ct Editing: AK, and NS. Visualization: AK. Supervision: NS.

Cite as:

Kara-Jovanović A, Suvajdžić-Vuković N. Clinical Presentation and Therapy of Primary Immune Thrombocytopenia Resistant to Splenectomy. Int J Med Students. 2020 Jan-Apr;8(1):11-14.

This work is licensed under a Creative Commons Attribution 4.0 International License

ISSN 2076-6327

This journal is published by the University Library System, University of Pittsburgh as part of the Digital Publishing Program and is co-sponsored by the University of Pittsburgh Press. 